\title{
Technology Tools for Working or Teaching Remotely
}

\author{
Jon Eckhardt (University of Wisconsin-Madison)
}

\section{KEYWORDS: Teaching Methods, crisis management.}

While many in the business world are accustomed to working remotely, many teachers feel online instruction and meetings will never replace being in the same classroom together. However, companies have created online communication platforms and supporting technologies that can keep businesses humming and students learning even when being physically in the same place is not possible.

While they require an adjustment, in some cases these online technologies provide capabilities that do not exist in a bricks and mortar setting, much like online journals allow for comments when paper journals don't.

Below is an overview of Software as a Service (SAAS) business technologies that can be used both in a business and classroom setting.

-

Slack (slack.com) - Slack is a messaging system designed to facilitate communication among business teams. A team of individuals subscribes to a slack workspace. Within the workspace are conversations, named channels, with each channel for a specific topic. A channel can be either private or public, with private channels only visible to some within a workspace and public channels being visible to all members of the workspace. The workspace has an administrator, who sets policy (for example, deciding who belongs to each channel) and who can be a member of the workspace. If we have a class with team projects, the workspace would be a class and separate channels would then be created for inclass discussions, course administrative items, sharing news articles related to the class, and for each team project.

Course Management Systems - Canvas, Blackboard, and Moodle are online course management systems used by many colleges and universities. Many of these platforms have the ability to give and automatically grade quizzes and exams. I have two suggestions regarding online exams. First, make the exams open book but hard to complete in a short period of time unless the student studied. Then, use the time limit feature in the course management system to put a limit on the time available for the student to take the exam. Second, give the exam during your regular class period, using a video conference platform so you are available to take questions.

Zoom (zoom.com) - Zoom is an online video conferencing tool that was designed to be more intuitive to use for first-time users than other online conferencing tools, while also including advanced features. In the classroom, Zoom has features that can be used to manage in-class discussions (called breakout rooms), which can be an effective way to teach online. In addition to hosting classes and lectures, I have used Zoom to give exams remotely. If you put each student in their own breakout room, they can ask questions about the exam without disturbing other students.

Calendly (calendly.com) - Calendly is a scheduling tool that, when connected with Zoom, can help you automatically schedule online video calls. You can use it for your business or with your students for office hours. In Calendly you can set up times when you are available for meetings, and then share a link with meeting participants. When someone schedules an appointment, Calendly will create the Zoom link, email the people involved, and create a calendar event on your calendar with all of the meeting details. This is a real time saver! 
Email - Everyone knows how to use email. Students do tend to respond to emails from faculty, especially if you include your course number and a compelling phrase in your subject line.

-

Airtable (airtable.com) - Airtable is an easy to use, high-powered relational database system. I use Airtable to keep records of all the interactions I have with my students, as well as custom assignments I give to each team in my class.

Zapier (zapier.com) - Zapier is a software tool that gives you the power of programming without being a programmer. Zapier allows you to powerfully connect different SAAS products. For example, you can use Zapier to pull discussion from Slack into Airtable so you can track comments made by each student on Slack to help you correctly credit students with discussion points. Zapier is a great way to impress your friends with your computing prowess!

We hope you find these tools helpful as you shift to remote working or teaching.

Image by Tumisu (https://pixabay.com/users/Tumisu-148124/?utm_sourc e=link-attribution\&utm_medium=referral\&utm_campaig n=image\&utm_content=4727942) from Pixabay (https://pixabay.com/?utm_source=link-attribution\&utm _medium=referral\&utm_campaign=image\&utm_content $=4727942$ ) 